\title{
METODOLOGÍA PARA EL DESARROLLO DE CURSOS EN LA MODALIDAD DE M-LEARNING A TRAVÉS DE MENSAJERÍA INSTANTÁNEA
}

METHODOLOGY FOR THE DEVELOPMENT OF COURSES IN THE FORM OF M-LEARNING THROUGH INSTANT MESSAGING

CARLOS BRAVO REYES FÁTIMA APAZA ZEGARRA JAVIER OROZCO ALDANA

UNIVERSIDAD AUTÓNOMA GABRIEL RENÉ MORENO -UAGRM BOLIVIA 


\title{
RESUMEN
}

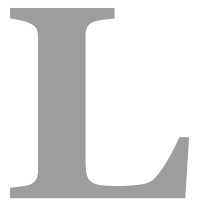

as redes sociales están cada vez más presentes en todas las actividades humanas y en especial en las vinculadas a la enseñanza. Pero su presencia no es garantía de su utilidad, es la organización pedagógica el elemento que distingue en gran parte el éxito o el fracaso de las actividades de aprendizaje. El trabajo describe la metodología empleada en el desarrollo de un sistema de cursos en línea, a partir de la concepción del micro aprendizaje y aplicada a la mensajería instantánea. Se empleó la red social WhatsApp para verificar la propuesta metodológica a partir de siete cursos desarrollados con participantes de más de 13 naciones.

Palabras Clave: m-learning; micro-aprendizaje; micro contenidos; wooc; aprendizaje; redes sociales; whatsapp.

\begin{abstract}
Social networks are increasingly present in all human activities and especially those related to education. But their presence does not guarantee its usefulness; educational organization is the element that distinguishes much of the success or failure of the learning activities. The paper describes the methodology used in the development of a system of online courses, from the conception of learning and applied micro instant messaging. WhatsApp social network was used to verify the methodology developed from seven courses with participants from over 13 nations.
\end{abstract}

Keyword: m-learning; learning micro; micro content wooc; learning; social networking; whatsapp.

\section{INTRODUCCIÓN}

Los dispositivos móviles, como tabletas, portátiles y en particular los celulares denominados "Smartphone" forman parte de las últimas tecnologías que llegaron al aula. Su presencia es casi generalizada entre estudiantes y profesores, que transformaron a este medio en un recurso permanente en todas nuestras actividades.

Los celulares actuales facilitan el empleo de diversas tecnologías convergentes, lo que significa que desde el equipo se puede conectar al correo, navegar en Internet y acceder a las 
redes sociales, tal y como se realiza desde una computadora. Esta es una de las tantas posibilidades para introducir los celulares en el proceso pedagógico, donde el estudiante puede realizar tareas similares a las que ejecuta frente a la computadora.

Un segundo elemento a tomar en cuenta está referido a la movilidad del sujeto y del equipo, lo que mejora la realización de disímiles tareas en diferentes lugares y tiempos. Así el celular se transforma en un vehículo que facilita la información, la investigación y las actividades interactivas.

Cuando a todo lo anterior se le agregan las redes sociales nos encontramos con el potencial de resolver problemas de la vida real, de comunicar la información y los conocimientos del individuo y en especial de colaborar con otros en su proceso de aprendizaje.

La metodología que explicamos se apoya en la concepción del micro aprendizaje bajo la modalidad del m-learning a través de servicios de mensajería instantánea, en este caso WhatsApp, una de las redes de mayor crecimiento en el último año y utilizada por profesores y estudiantes de diferentes niveles educacionales.

\section{DESARROLLO}

\section{Los celulares en el ámbito educacional}

La inserción de las tecnologías en el proceso pedagógico es un fenómeno iniciado desde hace más de cien años. En los albores del pasado siglo el cine fue empleado en diferentes instituciones educacionales, mientras la radio se aprovechaba en variados procesos de capacitación, esto sin olvidar la presencia permanente del material impreso.

Pero el proceso de incorporación de la tecnología a la escuela, tuvo un despegue después de la Segunda Guerra Mundial, cuando la mayoría de los estados europeos acudieron a la tecnología para reconstruir sus destrozadas economías. Surge el criterio que la tecnología es obligada para el desarrollo acelerado de sus sistemas económicos, idea que se traslada a la educación. Es el momento en que aparece el conocido concepto de Tecnología Educativa, asociado de manera general al empleo de la tecnología.

El desarrollo tecnológico de nuestra época permitió la creación de Internet y sus infinitas posibilidades de desarrollo social. La Red favoreció la difusión de la información y esta a su vez comenzó a crecer de manera exponencial llegando a lo que se denomina infoxicación, derivado del término anglosajón "information overload" y acuñado por Alvin Toffler (1970) en su libro Future Shock.

En la actividad educativa este problema se manifiesta de manera permanente, los profesores exigen trabajos de investigación donde la totalidad de los estudiantes acude a Internet, la información es generalmente copiada y en muchas ocasiones con la ausencia de un análisis crítico. 
Pero la tecnología por sí sola no resuelve los problemas de aprendizaje que se encuentran en la enseñanza. Es necesaria una visión pedagógica de su empleo en el proceso pedagógico. La tecnología va muy por delante de su inserción en la enseñanza universitaria. Priman en muchas ocasiones las decisiones administrativas sobre la compra de medios, algunos de ellos pobremente empleados. Un ejemplo actual se aprecia en el uso de las pizarras digitales interactivas, empleadas principalmente como medio de proyección desaprovechando su potencial didáctico.

A lo anterior se agrega el alto costo de muchos medios que escasamente son aprovechados en todas sus posibilidades didácticas, en perjuicio de otras tecnologías que pueden ser usadas con un mínimo de gasto y un gran beneficio pedagógico.

Una manera de aprovechar al máximo la tecnología existente es la conocida metodología BYOD (bring your own device) que en castellano significa trae tu propia tecnología. Trasladada al proceso de enseñanza, supone que los estudiantes pueden traer y emplear en clases su propia tecnología.

Dentro de ellos están presentes los dispositivos móviles, como es el caso de los celulares, objeto de la experiencia. Como se comentó anteriormente los celulares son objetos cotidianos por parte de estudiantes y profesores y han llegado a modificar diferentes hábitos, desde las formas de acceder a la información hasta la de comunicarnos.

Todo tipo de tecnología tiene el potencial de ser incorporada a la actividad pedagógica, pero debe primar ante todo la necesidad de su inclusión, la metodología de su empleo y la relación con estudiantes y profesores. Su inserción adecuada puede favorecer la motivación, la seguridad y el interés.

\section{M_learning, el micro aprendizaje y los micro contenidos}

La existencia de diferentes medios fue la base técnica para el funcionamiento y posterior desarrollo de la educación a distancia. Su presencia permitió otras formas de organizar el aprendizaje a distancia, entre las que se encuentra el m-learning o aprendizaje con el empleo del móvil.

Borja (2012) concibe este tipo de organización como una forma de intersección de la computación móvil y el E-learning, y opina que se caracteriza por la capacidad de acceder a recursos de aprendizajes desde cualquier lugar, en cualquier momento, con alta capacidad de búsqueda, alta interacción y alto soporte para un aprendizaje efectivo.

Koole (2009) comenta que a pesar que las tecnologías móviles tienen un amplio empleo las definiciones en relación a su uso en la educación están limitadas a posiciones más técnicas que pedagógicas. En nuestro criterio esto se debe a la celeridad con la que se produce la inserción de las tecnologías y la poca investigación pedagógica en este campo. No es un fenómeno nuevo, se viene generando permanentemente a lo largo de la historia de la educación.

En su trabajo Koole (2009) se refiere al m-learning como la convergencia de tecnologías móviles, las capacidades de aprendizaje del ser humano y la interacción social. 
Romero y otros (2010) señalan que el aprendizaje móvil promete ser la tecnología educativa del nuevo siglo que de acceso frecuente e integral a los sistemas y aplicaciones que apoyan el aprendizaje formal e informal en cualquier momento y en cualquier lugar dando la oportunidad a las personas de controlar y aprovechar sus tiempos disponibles.

Molina (2010) cita que el aprendizaje móvil se caracteriza por su capacidad de entregar contenidos de aprendizaje sin fronteras de tiempo ni espacio a través de dispositivos móviles, tales como teléfonos celulares, agendas electrónicas, pequeñas computadoras y/o todo dispositivo de mano que tenga alguna forma de conectividad inalámbrica con la finalidad de maximizar los tiempos disponibles para el aprendizaje.

En nuestro criterio el m-learning es una forma de organización de la actividad pedagógica, aplicable a cualquier tipo de ambiente sea formal o no. Por tal razón lo más importante de esta forma es la manera en que se organiza el aprendizaje y no el medio a través del cual se acceda. Existe la tendencia a considerar que es un proceso de m-learning cuando se emplea el dispositivo móvil. Sin embargo debemos entender que no es el medio el elemento que define este tipo de aprendizaje, sino su forma de organizarlo. La integración de tecnología, permite también emplear los mismos recursos del celular en la computadora. Por ejemplo desde la computadora de escritorio tanto el profesor como los estudiantes pueden emplear WhatsApp, con solo disponer del navegador Google Chrome y la aplicación de escritorio de esta red.

Por esta razón consideramos que la manera adecuada de crear un verdadero proceso de m-learning es cuando partimos de la concepción de micro aprendizaje. Molina citando a Hug (2010) considera que este se organiza en términos de momentos o episodios especiales de aprendizaje que utilizan contenidos o tareas especiales dentro de pequeñas etapas. El término micro-aprendizaje se relaciona con micro contenidos y micro-medios.

El desarrollo de procesos de micro aprendizaje emplea (aunque no necesariamente) recursos móviles como es el caso de los celulares inteligentes o Smartphone. Ellos contribuyen como señalamos, a la convergencia digital y favorecen su empleo en las actividades de aprendizaje. El esquema sintetiza este proceso.

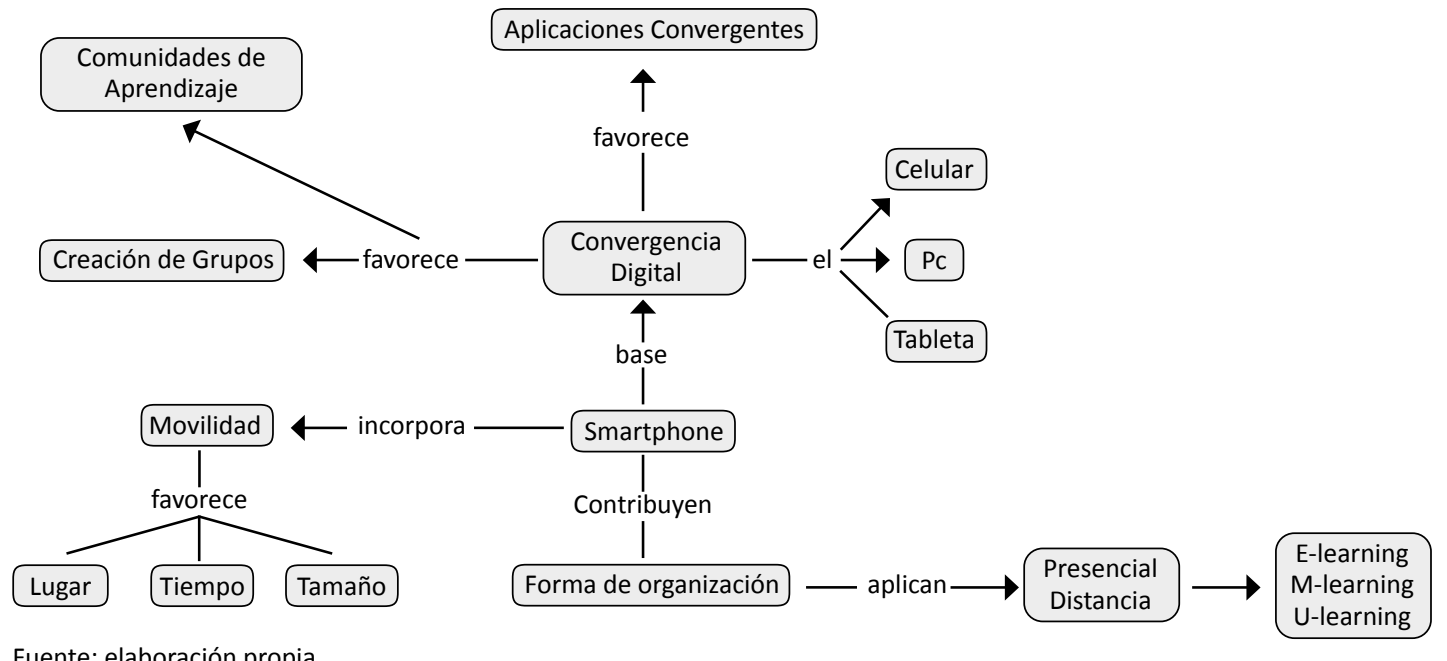

Fuente: elaboración propia 


\begin{tabular}{c}
\hline CARLOS BRAVO REYES - FÁTIMA APAZA ZEGARRA - JAVIER OROZCO ALDANA \\
METODOLOGÍA PARA EL DESARROLLO DE CURSOS EN LA MODALIDAD DE M-LEARNING... \\
Pags. 43 - 56
\end{tabular}

Como se señaló el proceso de micro aprendizaje toma en cuenta micro contenidos y micro medios. Los primeros se refieren a unidades autónomas de contenido, de carácter preciso y que su tratamiento requiera poco tiempo. No significa que el contenido sea tratado de manera superficial, sino que para la comprensión de su esencia se requiere de menos tiempo.

Uno de los errores frecuentemente encontrados en los medios empleados en el m-learning es que estos se crearon bajo la concepción de la enseñanza a distancia con el uso de medios como los documentos impresos, que se traspasaron directamente a medios digitales. Con esto los medios solamente migraron de formato, bajo la excusa de mantener su contenido.

Para minimizar estos errores en la metodología que describiremos a continuación partimos de un diseño tomando en cuenta que tanto contenidos como medios se desarrollarían por medio de dispositivos móviles y más específicamente un Smartphone.

\section{Metodología para el desarrollo de cursos con el empleo de WhatsApp. (Wooc)}

Como señalamos anteriormente las experiencias realizadas en cursos de m-learning fueron desarrolladas en WhatsApp. Esta es una red social creada por Jan Koum y Brian Acton en febrero del 2009 en California. A finales de ese mismo año se convirtió en la quinta aplicación más descargada para el sistema Android. En febrero del 2014 fue adquirida por Facebook por un valor de 19 billones de dólares, cifra muy superior a la que propuso Google.

Sus características son sencillas, se instala en un Smartphone, se pueden enviar mensajes de texto, sonoros, videos e imágenes, además de las llamadas entre usuarios de la Red. Crece a un promedio de un millón de usuarios cada día. Se pueden crear grupos de hasta cien personas y es posible acceder a la Red desde el navegador Google Chrome.

En promedio los usuarios de la Red dedican a la semana 195 min y eso permite que cada día se envíen 30 billones de mensajes de texto, 700 millones de fotografías y 100 millones de video.

El neologismo que estamos empleando (Wooc) parte del concepto de Mooc. Este significa Massive Open On line course, por lo que Wooc, representa WhatsApp Open On line Course. En otras palabras curso abierto, en línea con el empleo de WhatsApp.

La metodología para la realización de los Wooc la estructuramos en los siguientes componentes:

* Determinación del objetivo del curso

* Análisis de las características de la Red

* Diseño de los contenidos

* Diseño de los medios

* Determinación de las formas de interacción

* Evaluación de los resultados 
El esquema muestra la secuencia de los pasos seguidos en la metodología.

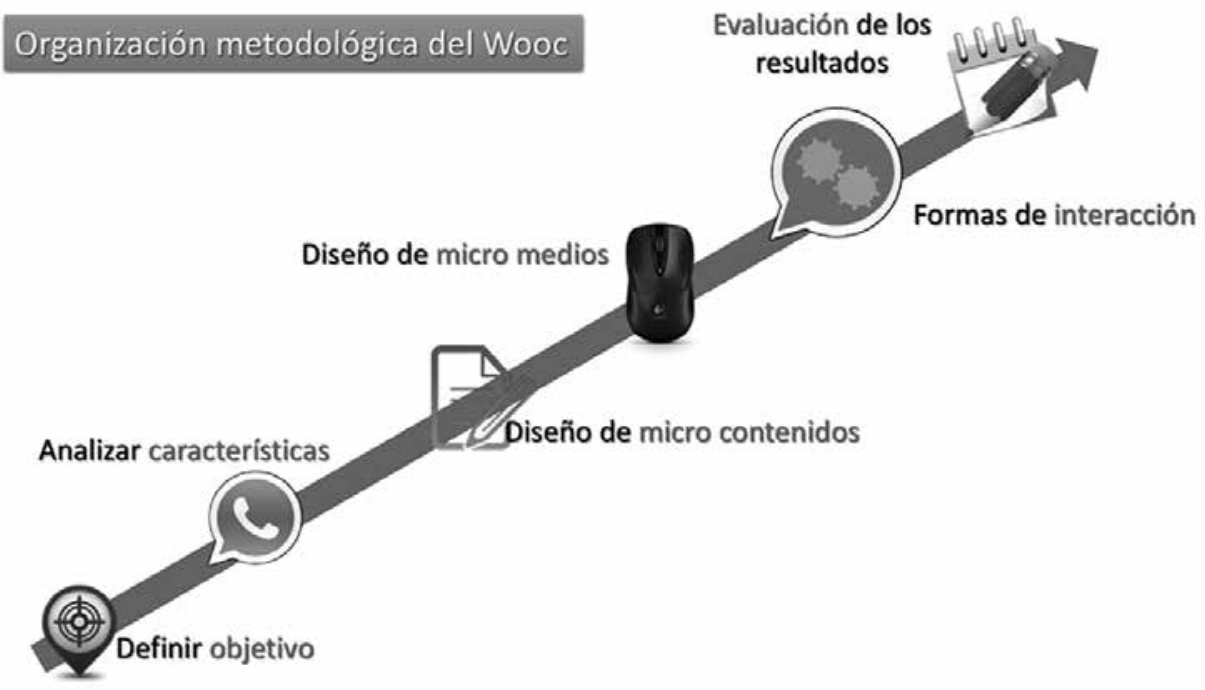

Fuente: elaboración propia

\section{Determinación del objetivo}

El punto de partida de la metodología es la determinación del objetivo del curso. Entre sus características destaca que debe ser puntual, tomar en cuenta el tipo de personas a las que va dirigido, el tiempo de duración del micro curso, el contenido a tratar y los medios a emplear. Se sugiere un único objetivo que permita el desarrollo de contenidos que sean asimilados con brevedad. No se trata de fragmentar el contenido, ni de minimizar su importancia, todo lo contrario. La actividad que se desarrolla implica una secuencia mínima de acciones, donde se vincula tanto el contenido teórico, como su puesta en práctica.

Por ejemplo un Wooc sobre la Segunda Guerra Mundial es imposible de desarrollar por la complejidad del tema y su amplitud, a la vez que consumiría mucho tiempo a los participantes, favoreciendo la pérdida de interés y por ende la no culminación del curso. Sin embargo abordar un momento particular de una batalla, la cifra de fallecidos, los países involucrados entre otros ejemplos, requieren de un breve tiempo y aseguran el cumplimiento del objetivo.

\section{Características de la Red}

El siguiente paso es revisar las características del sistema de mensajería instantáneo, que en nuestro caso fue WhatsApp y que como toda red de este tipo sufre modificaciones. En los últimos meses, los grupos pasaron de 50 a 100 personas y se incorporaron llamadas telefónicas. En la primera experiencia que realizamos los grupos solo permitían 50 personas por lo que fue necesario crear tres grupos, para atender a todos los matriculados. 


\section{Diseño de los micro contenidos}

La relación objetivo micro contenidos es clave en la organización del Wooc. Estos últimos deben ser puntuales y de gran precisión y siempre en correspondencia con el objetivo. Los contenidos deben favorecer el desarrollo práctico de las tareas y ser diseñados específicamente para este tipo de tarea.

\section{Diseño de los micro medios}

Con los datos anteriores el siguiente paso es la selección y diseño de los medios o recursos a emplear. Partimos nuevamente del criterio en la puntualidad de los contenidos por ello los recursos seleccionados deben favorecer una información clara, rápida de leer y atractiva. A esto se agrega que los medios tienen que ser diseñados tomando en cuenta tanto las características de la Red, como el tamaño de la pantalla de un Smartphone. En esto último existen variedad de tamaños, por lo que previamente se probaron en Smartphone de tamaños diferentes de pantalla. Tampoco se olvidó que algunas personas podían seguir el Wooc a través de sus equipos de escritorio.

También es necesario tomar en cuenta que las cápsulas se envían en una secuencia lógica y respetando el tiempo necesario para analizar el contenido y responder a preguntas que tienen el objetivo de generar el debate entre los participantes. En otras palabras los asistentes al micro curso deben tener una participación activa y no limitada a la lectura de las imágenes.

Siguiendo la lógica del micro aprendizaje que requiere de micro contenidos, estos a su vez se desarrollan sobre la base de micro medios. En el caso de la metodología preferimos emplear la categoría de "cápsulas informativas" en vez de micro medios.

Las imágenes corresponden a dos de las cápsulas informativas empleadas en el primer Wooc

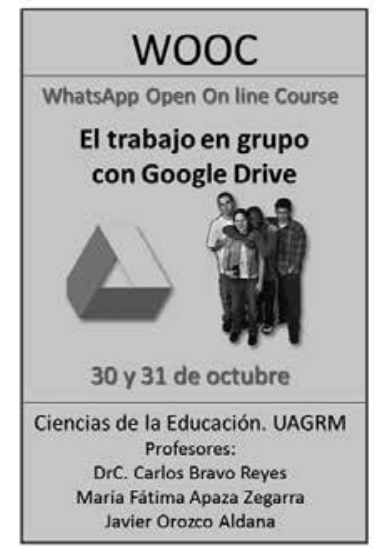

Fuente: elaboración propia

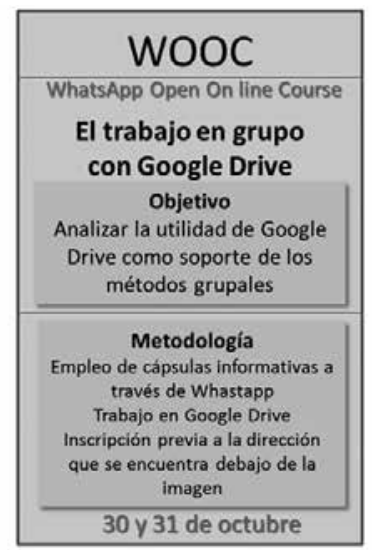

Fuente: elaboración propia

Como se observa las cápsulas tienen una elaboración sencilla, con textos cortos y precisos, 
además de emplear imágenes con alta claridad y de fácil decodificación. Las cápsulas cumplen diferentes funciones, entre ellas las de comunicar informaciones generales como reglas del grupo, el objetivo y la forma de trabajo, las de retroalimentación y evaluación entre las más importantes.

Las cápsulas que se muestran a continuación corresponden a otro de los Wooc que realizamos con el empleo de la metodología.

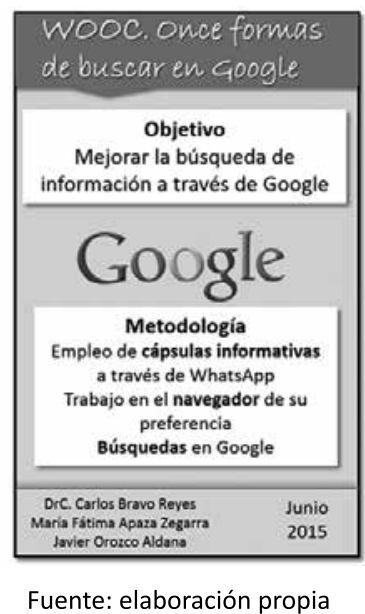

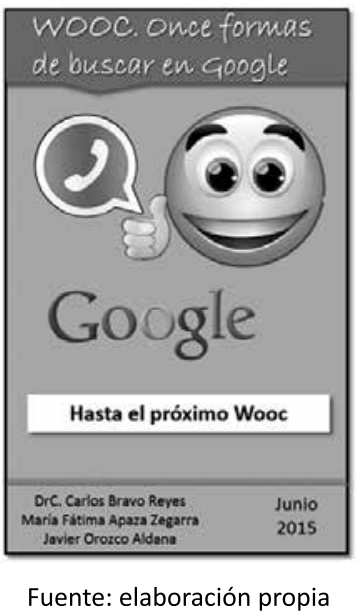

\section{Formas de interacción}

El siguiente paso se corresponde a prever las formas en que se interactúa con los participantes. Es necesario como en cualquier actividad establecer algunas reglas de trabajo, como el respeto a los contenidos, la responsabilidad por las opiniones propias y evitar la realización de comentarios ajenos al Wooc. En la elaboración de las cápsulas es necesario considerar que los participantes respondan a preguntas, expresen sus criterios y experiencias sobre el tema tratado, asumiendo una posición dinamizadora. La imagen muestra una cápsula empleada en otro micro curso donde se expone una pregunta para el debate, posterior a los contenidos tratados.

Es también importante reducir o evitar el envío de mensajes

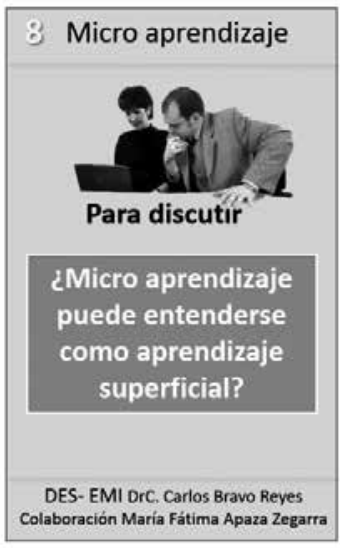

Fuente: elaboración propia en video y en especial enlaces a otros sitios, si estos no son absolutamente necesarios. Se debe recordar que el diseño de los medios obedece al concepto del micro medio y la inclusión de medios con más información puede perjudicar esta concepción.

También se debe considerar que muchas personas pueden disponer de conexiones a internet con baja velocidad o costos altos por el uso de estos servicios. 


\section{Evaluación de los resultados}

El último de los pasos en la metodología es la evaluación de los resultados, donde no se debe confundir la evaluación con la calificación. En nuestro caso nos limitamos a evaluar el trabajo en el Wooc y no así la asimilación de los contenidos.

En el primero de los Wooc participaron 123 personas procedentes de 11 países de América Latina más España.

Para medir el impacto del Wooc se respondió una encuesta en línea, donde en la primera de las preguntas, los participantes demostraron no haber participado con anterioridad en una experiencia de este tipo.

Participó con anterioridad en un curso de Whatsapp

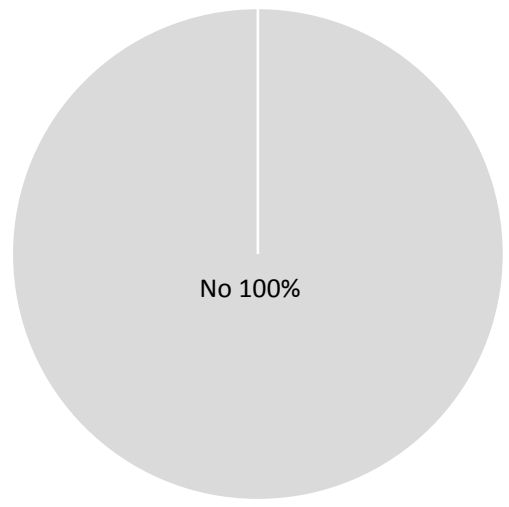

Fuente: elaboración propia

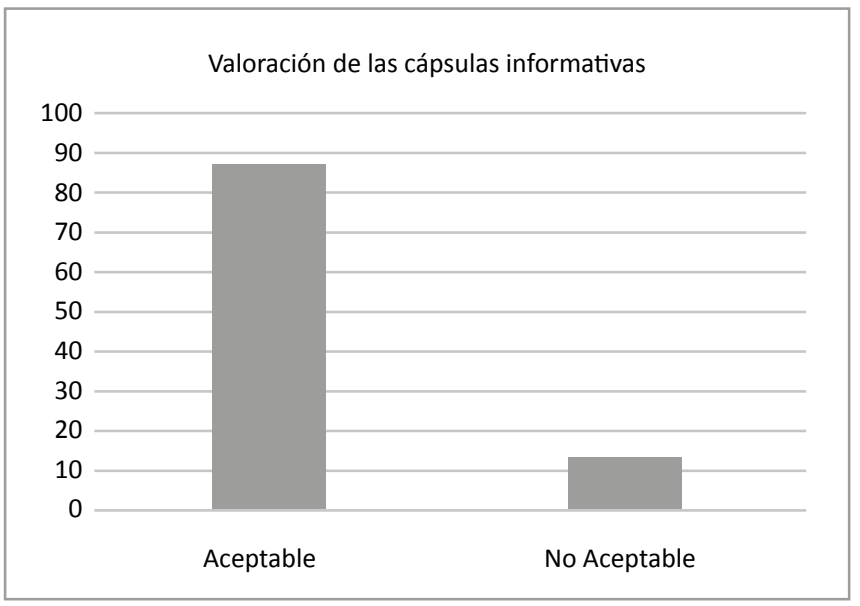

Fuente: elaboración propia

Otra de las preguntas indagó sobre la valoración de las cápsulas informativas, donde la mayoría de los participantes las evalúo como aceptables. 
Una tercera pregunta estuvo dirigida a la opinión sobre la metodología desarrollada en el Wooc, las respuestas se aprecian en la imagen.

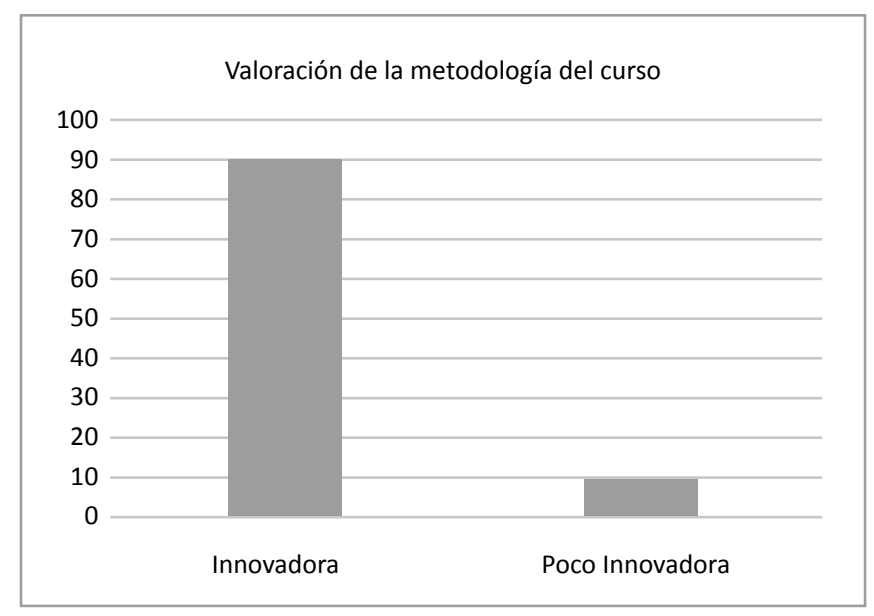

Fuente: elaboración propia

El desarrollo de estos Wooc fue posible gracias al trabajo de dos estudiantes de la licenciatura en Ciencias de la Educación de nuestra Universidad. Ellos coautores del artículo, trabajaron en todas las fases de la metodología, así como en otros Wooc. Hasta este momento nuestro equipo ha desarrollado siete Wooc, con la participación de más de 300 personas de 12 diferentes países.

\section{CONCLUSIONES}

El desarrollo de un curso bajo la forma de m-learning requiere de una organización didáctica por lo que debe seguir una secuencia propia del diseño instruccional. El Wooc es una manera de organizar el aprendizaje con el empleo de celulares inteligentes. Parte de la concepción del micro aprendizaje, desarrollado a través de micro contenidos.

Uno de los elementos claves en el desarrollo de cualquier acción formativa mediante el m-learning es concebir los medios para este tipo de actividad, tomando en cuenta el recurso a través del cual estos se desplegaran. En el caso de la metodología propuesta, las cápsulas informativas o micro medios, tomaron en cuenta que su empleo es a través de un celular. 


\section{REFERENCIAS BIBLIOGRÁFICAS}

Almonte Moreno, Mario G Micro contenidos en el Ámbito del aprendizaje http://aprendizajeenred.es/2013/10/30/microcontenidos-aprendizaje. Consultado en julio del 2015.

Bausela Herreras, Esperanza. “La docencia a través de la investigación acción”. Revista Iberoamericana de educación. 2012 Disponible en www.rieoei.org/deloslectores/682Bausela.PDF

Borja Martín Herrera. El móvil en la educación: un nuevo paradigma. Ventajas y desventajas de su uso. Tesis final de máster. Universidad de la Rioja. http://es.slideshare.net/ Yasnaya/2012-09-24tfmestudiodeltrabajo. Consultado en julio del 2015.

Bravo Reyes, Carlos. Innova Educa 2015 y el desarrollo de un Wooc. http://366-dias.blogspot. com/2015/o6/innova-educa-2015-y-el-desarrollo-de-un.html. Consultado en julio del 2015.

Bravo Reyes, Carlos. WhatsApp Open Online Course (Wooc) o un curso con el empleo de Whatsapp. Publicado en: http://366-dias.blogspot.com.ar/ Consultado en julio del 2015.

Fontas, Carina. La técnica de los grupos focales en el marco de la investigación socio cualitativa. En: http://www.fhumyar.unr.edu.ar/escuelas/3/materiales\%20de\%20catedras/ trabajo\%20de\%20campo/profesoras.htm Consultado en julio del 2015.

Gómez, Ignacio. El increíble vídeo menguante y la necesidad de definir nuevas estrategias de contenido en el móvil” En: http://innovacionaudiovisual.com/2015/03/o6/el-increiblevideo-menguante-y-la-necesidad-de-definir-nuevas-estrategias-de-contenido-en-el-movil/ Consultado en julio del 2015.

Koole, M y otros. (2009). “A Model for Framing Mobile Learnig,”Mobile Learning Transforming the Delivery of Education and Training Athabasca University. Disponible: http:// www.aupress.ca/ Consultado en julio del 2015.

Molina Martín José, Romero. Ambiente de Aprendizaje Móvil Basado en Micro-Aprendizaje. Revista Iberoamericana de Tecnologías del Aprendizaje. IEEE-RITA Vol. 5, Núm. 4, Nov. 2010.

Prensky, Marc: Nativos e Inmigrantes Digitales. Institución educativa SEK. Disponible en http://www.marcprensky.com/writing/Prensky-NATIVOS\%20E\%20INMIGRANTES\%20 DIGITALES\%20\%28SEK\%29.pdf Consultado en julio del 2015. 
Romero David, Molina Arturo, Chirino Violeta. Aprendizaje Móvil: Tendencias, Cuestiones y Retos" Revista Iberoamericana de Tecnologías del Aprendizaje. IEEE-RITA Vol. 5, Núm. 4, Nov. 2010.

Sánchez Aparicio, Juan Carlos. El Microlearning está en todas partes” http://www.snackson.com/el-microlearning-esta-en-todas-partes-10-ejemplos Consultado en julio del 2015.

Santamaría Gonzales, Fernando. Microlearning microaprendizaje: definiciones y características http://fernandosantamaria.com/blog/tag/microcontenido Consultado en julio del 2015.

Toffler, Alvin. El shock del futuro. Plaza \& Janes Editores. Barcelona 1970.

\section{CURRICULUM VITAE}

\section{Carlos Bravo Reyes}

Lic. en Educación y Doctor en Ciencias Pedagógicas por la Comisión de Grados Científicos de Cuba. Distinción nacional por la educación en Cuba.

Profesor Titular de Tecnología educativa en la Facultad de Humanidades de la UAGRM.

Asesor pedagógico de la Dirección de Tecnología educativa y educación a distancia de la UAGRM. Profesor de posgrado en varias universidades de Cuba, Bolivia y de la Escuela Politécnica Nacional de Quito. Impartió más de 100 posgrados en el campo de la educación. Ex director del programa de formación de Doctorado en Ciencias de la Educación de la UAGRM.

Miembro del Comité editorial y comité científico de varias revistas científicas (RED, RIED. RFCE, Apertura y otras). Autor de varios libros y artículos científicos.

Escribe con frecuencia sobre temas educacionales en su blog 366-dias.blogspot.com.ar cbravo@catedradigital.info

\section{Fátima Apaza Zegarra}

Asistente de la Dirección de Tecnología educativa y educación a distancia de la UAGRM. Licenciada en Ciencias de la Educación.

Diplomas obtenidos: Estrategias y habilidades para una comunicación efectiva.

$2^{\text {das. }}$ Jornadas de actualización y evaluación de las TIC en la Educación.

Participante en el Curso Masivo, Abierto y en Línea "Tecnología Educativa a través de las Redes Sociales".

Investigación premiada en la ExpoCiencia 2015 Libro impreso multimedia con códigos Qr y Realidad Aumentada sobre el sistema personal de aprendizaje. 
Facilitadora en el Curso masivo, abierto y en línea "La presentación perfecta en Power Point no existe, pero podemos acercarnos".

apazazegarrafatima@gmail.com

\section{Javier Orozco Aldana}

Asistente de la Dirección de Tecnología educativa y educación a distancia de la UAGRM. Especialista en Hardware y Software.

Tutor en el Mooc "Tecnología educativa a través de las redes sociales". Primer premio de la ExpoCiencia 2014 de la UAGRM.

Tutor del Mooc "Evernote, un recurso indispensable para la enseñanza".

Facilitador del Mooc "La presentación perfecta no existe pero podemos acercarnos".

Asistente Digital, de la Facultad de Humanidades.

Actualmente culmina la Licenciatura en Ciencias de la Educación.

javito.o.a@gmail.com 